\title{
Inhibitory Effects of Methanol Extracts of Herbal Medicines on Substance P-Induced Itch-Scratch Response
}

\author{
Chihiro Tohda, ${ }^{a}$ Yumiko Kakihara, ${ }^{b}$ Katsuko Komatsu, ${ }^{a}$ and Yasushi Kuraishi ${ }^{*}, b$ \\ Research Center for Ethnomedicines, Institute of Natural Medicine, ${ }^{a}$ and Department of Applied Pharmacology, Faculty of \\ Pharmaceutical Sciences, ${ }^{b}$ Toyama Medical and Pharmaceutical University, 2630 Sugitani, Toyama 930-0194, Japan. \\ Received November 8, 1999; accepted January 11, 2000
}

In a search for new anti-pruritic drugs we screened methanol extracts of 33 herbal medicines which have been used for cutaneous diseases for their antipruritic activity using substance P (SP) as a pruritogen in mice. When administered perorally 30 min before SP injection, methanol extracts of 6 of these herbal medicines, the root of Scrophularia ningpoensis Hemst., the root of Patrinia villosa (Thunb.) Juss, the fruit of Forsythia suspenna VAHL, the rhizome of Cimicifuga dahurica (TURCZ.) MAXIM., the aerial part of Schizonepeta tenuifolia BrIQ. and the fruit of Cnidium monnieri (L.) Cuss, inhibited SP-induced itch-scratch response at a dose of $200 \mathrm{mg} / \mathrm{kg}$ without affecting locomotor activity. Dose dependence of these 6 extracts $(50-500 \mathrm{mg} / \mathrm{kg})$ was investigated and all of them inhibited SP-induced itch-scratch response, with extracts from Scrophularia ningpoensis, Schizonepeta tenuifolia and Cnidium monnieri showing particularly significant inhibition. The results suggest that these 6 methanol extracts have inhibitory activity against SP-induced itching.

Key words substance P; itch-scratch response; herbal medicine; mouse

Itching is the most common symptom of a cutaneous disease (e.g., atopic dermatitis, contact dermatitis and urticaria) and accompanies several systemic disorders (e.g., chronic renal failure and cholestasis). This unpleasant sensation stimulates scratching of the lesioned skin thus worsening the lesions. Although anti-histamine and anti-allergy drugs are generally prescribed for cutaneous pruritus, they do not allay a severe itching sensation. ${ }^{1,2)}$ Corticosteroids are also used as external medicines to relieve cutaneous pruritus, however, they are thought to produce an antipruritic effect as a result of their anti-inflammatory action and are therefore not applied to pruritus unless inflammation is present. Thus, the development of novel anti-pruritic drugs is necessary.

There are many herbal medicines which have been applied to cutaneous diseases and several Kampo formulae (the traditional Chinese and Japanese herbal medicines) are prescribed for dermatitis. In some cases they ameliorate the dermatitis, but their underlying mechanisms are not understood and scientific experiments devoted to the anti-pruritic effects of herbal medicines have been few and far between.

The purpose of this study was to find herbal medicines with antipruritic activity. We have found that substance $P$ (SP), one of the most potent pruritogenic endogenous peptides in human subjects, ${ }^{3)}$ elicits scratching of the treated skin by the hind paws in the mouse ${ }^{4)}$ and that SP-induced scratching in mice has features like those of human itching. ${ }^{5)}$ Therefore, we screened for herbal medicines with inhibiting activity to an SP-induced itch-scratch response in mice.

\section{MATERIALS AND METHODS}

Preparation of Methanol Extracts of Herbal Medicines Herbal medicines used in this study are listed in Table 1; all were purchased from Tochimoto Tenkaido Co., Ltd. (Osaka, Japan). Voucher specimens of each sample were deposited in the Museum of Materia Medica, Research Center for Ethnomedicines. Fifty grams of each herbal medicine was extracted with $300 \mathrm{ml}$ of methanol under the reflux condition for $3 \mathrm{~h}$. The decoction was evaporated under a reduced pres- sure and freeze-dried. These extracts were dissolved in 5\% arabic gum immediately before use.

Animals Male ICR mice (Japan SLC, Ltd., Shizuoka, Japan) 5 to 11 weeks of age were used. They were housed under controlled temperature $\left(23-25^{\circ} \mathrm{C}\right)$ and light (lights on from 08:00 to 20:00). Food and water were freely available.

Behavioral Observation Before behavioral recording, the mice ( 4 animals per observation) were put into an acrylic cage $(26 \times 18 \times 30 \mathrm{~cm})$ composed of four cells $(13 \times 9 \times 30 \mathrm{~cm})$ for $1 \mathrm{~h}$ for acclimation. Methanol extract $(100 \mu \mathrm{l})$ of herbal medicine was administered perorally (p.o.) and 30 min later SP (Peptide Institute, Minoh, Japan) was injected intradermally (i.d.) in $50 \mu$ physiological saline into the rostral part of the back. ${ }^{5)}$ Immediately after i.d. injection of SP, the mice were put back to the same cell and their behaviors were videotaped using an 8 -mm video camera for 20 min, with any experimenter outside the animals' room. Play back of the video allowed counting of the scratching behavior. Mice generally scratched several times with the hind paws for about $1 \mathrm{~s}$ and a series of these movements was counted as one bout of scratching. ${ }^{4)}$ The degree of inhibition of scratching was calculated based on the number of scratches per $20 \mathrm{~min}$ with the average number of the control group being $100 \%$. The locomotor activity was measured for $20 \mathrm{~min}$ by a locomotor activity sensor (Neuroscience Inc., Tokyo, Japan) from 30 min after extract administration.

Data Analysis Data were analyzed by Student's $t$-test or one-way analysis of variance and post hoc Dunnett's test; $p<0.05$ was considered as significant. The means of data are presented together with S.E.

\section{RESULTS}

When injected i.d. into the rostral back, SP $(100 \mathrm{nmol} / \mathrm{site})$ elicited scratching of the skin around the injected site by the hind paws. The effect of SP reached maximum at $100 \mathrm{nmol} / \mathrm{site}$, peaked in the initial 10-min period and had almost subsided by $20 \mathrm{~min}$ after the injection. ${ }^{5)}$ Therefore, SP $(100 \mathrm{nmol} / \mathrm{site})$-induced scratching was counted for $20 \mathrm{~min}$ 
Table 1. Effects of Methanol Extracts of Herbal Medicines on SP-Induced Itch-Scratch Response in Mice

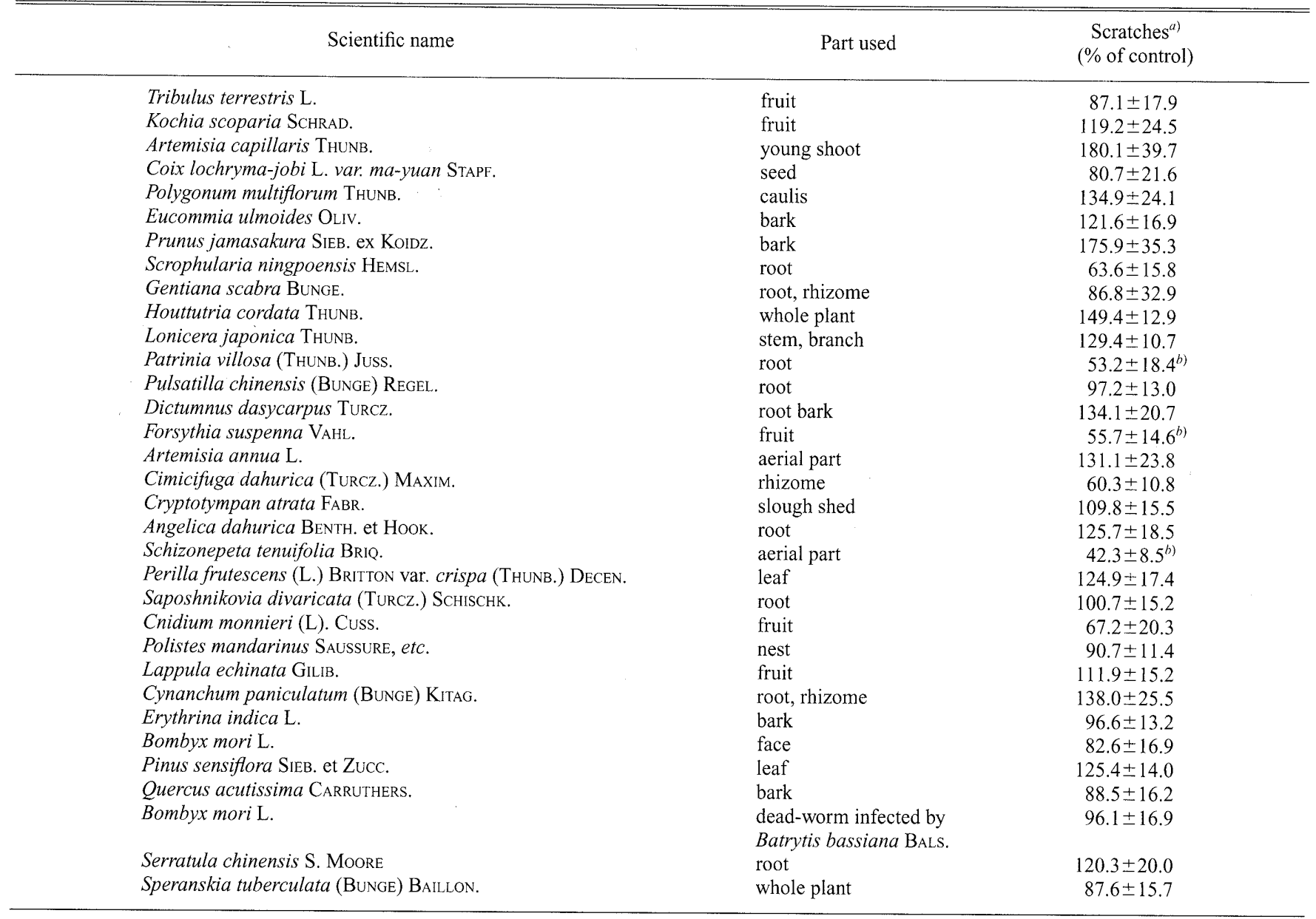

a) The number of scratchings of vehicle was $76.1 \pm 13.7$ per $20 \min (n=48) . b) p<0.05$ compared with vehicle control.

after i.d. injection. Among methanol extracts of 33 herbal medicines examined, 6 extracts from the root of Scrophularia ningpoensis HemsL., the root of Patrinia villosa (ThunB.) Juss, the fruit of Forsythia suspenna VAHL, the rhizome of Cimicifuga dahurica (TurCz.) MAXIM., the aerial part of Schizonepeta tenuifolia BRIQ. and the fruit of Cnidium monnieri (L.) Cuss inhibited SP-induced itch-scratch response to less than $70 \%$ of control at a p.o. dose of $200 \mathrm{mg} / \mathrm{kg}$ (Table 1); the inhibitory effects of Patrinia villosa, Forsythia suspenna and Schizonepeta tenuifolia were particularly significant.

These 6 methanol extracts did not significantly inhibit locomotor activity at a p.o. dose of $200 \mathrm{mg} / \mathrm{kg}$, and Scrophularia ningpoensis extract, in fact, tended to increase locomotor activity (Fig. 1).

Investigation of the dose-dependency of inhibitory action of the 6 extracts on SP-induced itch-scratch response (Fig. 2) showed that inhibition by the extract of Scrophularia ningpoensis at p.o. doses of 200 and $500 \mathrm{mg} / \mathrm{kg}$ was significant (Fig. 2A). The extract of Schizonepeta tenuifolia at p.o. doses of 50,200 and $500 \mathrm{mg} / \mathrm{kg}$ dose-dependently inhibited the response (Fig. 2E), while that of Cnidium monnieri (50$500 \mathrm{mg} / \mathrm{kg}$, p.o.) also dose-dependently inhibited the response, with almost complete inhibition at a dose of $500 \mathrm{mg} / \mathrm{kg}$ (Fig. 2F).

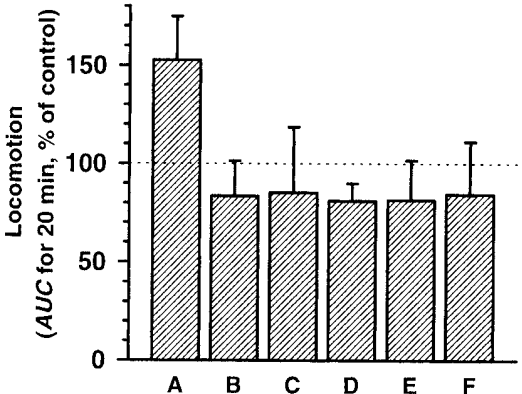

Fig. 1. Effects of Methanol Extracts on Locomotor Activity in Mice

Methanol extracts of the root of Scrophularia ningpoensis (A), the root of Patrinia villosa (B), the fruit of Forsythia suspenna (C), the rhizome of Cimicifuga dahurica (D), the aerial part of Schizonepeta tenuifolia (E) and the fruit of Cnidium monnieri (F) were p.o. administered at a dose of $200 \mathrm{mg} / \mathrm{kg}$. Thirty minutes after the administration, the number of locomotions was counted for $20 \mathrm{~min}$ and the area under the curve $(A U C)$ was calculated. Each value represents the mean \pm S.E. $(n=8) .{ }^{*} p<0.05$ when compared with control.

\section{DISCUSSION}

In the present experiments, from among 33 herbal medicines, methanol extracts from the root of Scrophularia ningpoensis, the root of Patrinia villosa, the fruit of Forsythia suspenna, the rhizoma of Cimicifuga dahurica, the aerial part of Schizonepeta tenuifolia and the fruit of Cnidium monnieri inhibited SP-induced scratching response in mice. Extracts of Scrophularia ningpoensis, Schizonepeta tenuifolia and Cnidium monnieri showed clear dose-dependent inhibition of the scratching. None of the 6 inhibited locomotor ac- 


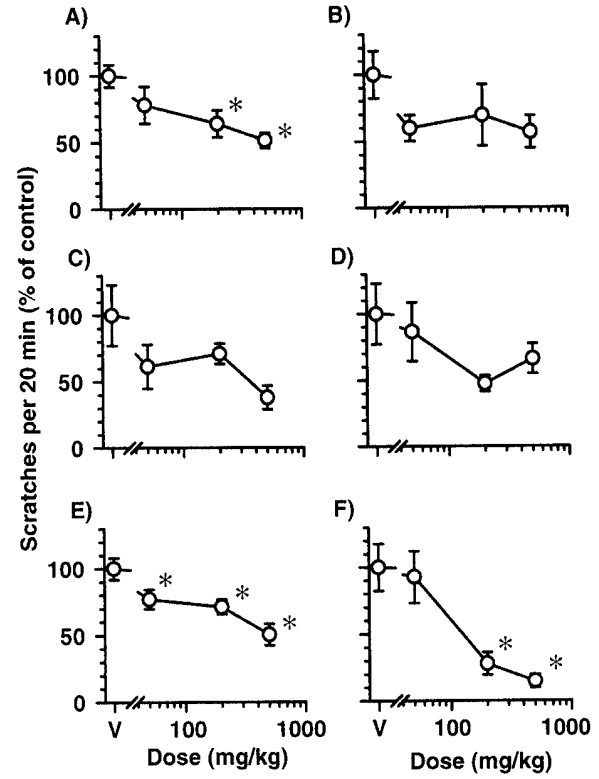

Fig. 2. Dose-Response Relationship of Inhibiting Action of Methanol Extracts on SP-Induced Itch-Scratch Response in Mice

The methanol extracts of Scrophularia ningpoensis (A), Patrinia villosa (B), Forsythia suspenna (C), Cimicifuga dahurica (D), Schizonepeta tenuifolia (E) and Cnidium monnieri (F) were administered p.o. at doses of 50,200 and $500 \mathrm{mg} / \mathrm{kg} 30 \mathrm{~min}$ before i.d. injection of SP $(100 \mathrm{nmol} / \mathrm{site})$. Immediately after the SP injection, the number of scratchings was measured for $20 \mathrm{~min}$. $\mathrm{V}$ on the $\mathrm{x}$-axis means vehicle administration (control). Each value represents the mean \pm S.E. $(n=8) .{ }^{*} p<0.05$ compared with control.

tivity at $200 \mathrm{mg} / \mathrm{kg}$, a dose which was effective against SP-induced scratching. Therefore, the scratch-inhibiting action of these extracts may be due to their inhibition of the itching sensation and/or scratching reflex, rather than to sedation or depression of general functions of the central nervous system. Since all extracts were administered $30 \mathrm{~min}$ before the test, we cannot exclude the possibility that some methanol extracts other than the 6 effective ones might inhibit SP-induced scratching with different timing of the pretreatment. However, as 17 extracts increased rather than decreased the scratching and the other 10 had almost no effect, the inhibitory action of these extracts if any may not be strong.

Repeated pretreatment with capsaicin, which alleviated human itching, also significantly inhibits SP-induced scratching in mice. ${ }^{5}$ SP-induced scratching is markedly inhibited by the opioid antagonist naloxone, ${ }^{5)}$ which ameliorates the itching sensation and scratching by patients with pruritic disease. SP is claimed to be involved in inflammatory responses, but the onset of the scratching behavior was immediate after SP injection, and no eczema or rash was seen within 20 min after injection in the mouse. These findings taken together suggest that SP-induced scratching is an itch response, and is not due to cutaneous inflammation. Thus, we suggest that the methanol extracts of the 6 herbal medicines referred to above inhibit the SP-induced itch sensation in the mouse. The water extracts of Patrinia villosa, Schizonepeta tenuifolia and Cnidium monnieri, but not those of Scrophularia ningpoensis, Forsythia suspenna or Cimicifuga dahurica, also significantly inhibited SP-induced itch-scratch response (unpublished observation). Thus, the anti-pruritic constituents of the first three may be extracted into both water and methanol.

Forsythia suspenna, Schizonepeta tenuifolia and Cimicifuga dahurica are included in several Kampo formulae (e.g., Keigai-rengyo-to, Bofu-Tsusho-san, Chizuso-ippo, Shoma-kakkon-to) which are clinically prescribed for cutaneous diseases such as atopic dermatitis. An ether fraction of a methanol extract of Cimicifuga dahurica ${ }^{6)}$ and a $70 \%$ methanol extract of Forsythia suspenna ${ }^{7)}$ inhibit carrageenaninduced edema in rats. Total coumarins in the fruit of Cnidium monnieri show a protective effect against histamine-induced bronchial asthma in guinea pigs. ${ }^{8)}$ These herbal medicines are thought to inhibit inflammation, but our results suggest that they have direct anti-pruritic potencies.

The mechanism of inhibiting actions of the herbal medicines on SP-induced itch-scratch response are unknown. However, the response by SP of the mouse is mediated at least partly by cutaneous $\mathrm{NK}_{1}$ receptors ${ }^{5)}$; mast cell-histamine systems are not involved. ${ }^{4,5)} \mathrm{SP}$ was reported to be increased in the lesioned skin of patients with pruritic dermatitis, such as atopic dermatitis ${ }^{9-11)}$ and psoriasis. ${ }^{12-14)}$ These findings suggest that SP in the skin is involved in some pruritic diseases. We need to investigate whether the sites of anti-pruritic effects of the methanol extracts are the skin or the central nervous system. To determine whether the antipruritic effects of methanol extracts of the herbal medicines are specific to SP, we are studying the inhibitory actions of the extracts on the itch-scratch response to serotonin ${ }^{15)}$ and histamine ${ }^{16)}$ of the mouse. We are also now trying to isolate active constituents from these methanol extracts.

Acknowledgement This work was supported in part by a Grant-in-Aid of Scientific Research (B) from the Ministry of Education, Science, Sports and Culture of Japan.

\section{REFERENCES}

1) Wahlgren C. F., Acta Dermato-Venereologica. Suppl., 165, 1-53 (1991).

2) Berth-Jones J., Graham-Brown R. A., Br. J. Dermatol., 121, 635-637 (1989).

3) Hägermark Ö., Hökfelt T., Pernow B., J. Invest. Dermatol., 71, 233235 (1978).

4) Kuraishi Y., Nagasawa T., Hayashi K., Satoh M., Eur. J. Pharmacol, 275, 229-233 (1995)

5) Andoh T., Nagasawa T., Satoh M., Kuraishi Y., J. Pharmacol. Exp. Ther, 286, 1140-1145 (1998).

6) Shibata M., Yamatake Y., Amagaya Y., Fukushima M. (abstract in English), Yakugaku Zasshi, 95, 539-546 (1975).

7) Ozaki Y., Rui J., Tang Y., Satake M., Biol. Pharm. Bull., 20, $861-864$ (1997).

8) Chen Z., Duan X. (abstract in English), Chung-Kuo Chung Yao Tsa Chih-China (J. Chinese Materia Medica), 15, 304-320 (1990).

9) Ostlere L. S., Cowen T., Rustin M. H. A., Clin. Exp. Dermatol., 20, 462-467 (1995).

10) Tobin D., Nabarro G., de la Faille H. B., van Voten W. A., van der Putte S. C. J., Schuueman H.-J., J. Allergy Clin. Immunol., 90, 613620 (1992).

11) Pincelli C., Fantini F., Massimi P., Girolomoni G., Seidenari S., Giannetti A., Br. J. Dermatol., 122, 745-750 (1990).

12) Chen J., Smoller B. R., Raychauduri S. P., Jiang W.-Y., Farber E. M., Arch. Dermatol. Res., 289, 611-616 (1997).

13) Al'abadie M. S. K., Senir H. J., Bleehen S. S., Gawkrodger D. J., Clin. Exp. Dermatol., 20, 384-389 (1995).

14) Eedy D. J., Johnston C. F., Shaw C., Buchanan K. D., J. Invest. Dermatol., 96, 434-438 (1991).

15) Yamaguchi T., Nagasawa T., Satoh M., Kuraishi Y., Neurosci. Res., 35, $77-83$ (1999).

16) Kitagawa K., Andoh T., Nagasawa T., Satoh M., Kuraishi Y., Jpn. J. Pharmacol., 73 (Suppl. 1), 93P (1997). 